\title{
The metabolic profile of mitoxantrone and its relation with mitoxantrone-induced cardiotoxicity
}

\author{
Luciana Grazziotin Rossato - Vera Marisa Costa - Paula Guedes de Pinho • \\ Marcelo Dutra Arbo • Victor de Freitas · Laure Vilain • \\ Maria de Lourdes Bastos · Carlos Palmeira $\cdot$ Fernando Remião
}

Received: 9 November 2012/Accepted: 8 March 2013/Published online: 2 April 2013

(c) Springer-Verlag Berlin Heidelberg 2013

\begin{abstract}
Mitoxantrone (MTX) is an antitumor agent that causes cardiotoxicity in $18 \%$ patients. The metabolic profile of MTX was assessed after incubation of $100 \mu \mathrm{M}$ MTX with hepatic S9 fraction isolated from rats. The presence of MTX and its metabolites was also assessed in vivo through the analysis of liver and heart extracts of MTX-treated rats. The cytotoxic effects of MTX and MTX metabolites were evaluated in the H9c2 cells after 24-h incubation with MTX alone and MTX + metabolites. The influence of CYP450- and CYP2E1-mediated metabolism for the cytotoxicity of MTX was assessed after 96-h incubation with MTX $(100 \mathrm{nM}$ and $1 \mu \mathrm{M})$ in the presence/ absence of CYP450 or CYP2E1 inhibitors. After 4-h incubation in supplemented S9 fraction, the MTX content was $35 \%$ lower and 5 metabolites were identified: an acetoxy ester derivative (never described before), two
\end{abstract}

Electronic supplementary material The online version of this article (doi:10.1007/s00204-013-1040-6) contains supplementary material, which is available to authorized users.

L. G. Rossato $(\bowtie)$. V. M. Costa · P. G. de Pinho .

M. D. Arbo · L. Vilain · M. de Lourdes Bastos · F. Remião ( $\square)$

REQUIMTE, Laboratório de Toxicologia, Departamento de Ciências Biológicas, Faculdade de Farmácia, Universidade do Porto, Rua Jorge Viterbo Ferreira, 228, Porto 4050-313, Portugal e-mail: luciana.g.rossato@gmail.com

F. Remião

e-mail: remiao@ff.up.pt

V. de Freitas

Centro de Investigação em Química, Departamento de Química,

Faculdade de Ciências, Universidade do Porto, Porto, Portugal

C. Palmeira

Centro de Neurociências e Biologia Celular de Coimbra, Departamento de Ciências da Vida, Universidade de Coimbra, Coimbra, Portugal glutathione conjugates, a monocarboxylic acid derivative, and the naphtoquinoxaline, the later commonly related to MTX pharmacological effects. The presence of MTX and naphtoquinoxaline metabolite was evidenced in vivo in liver and heart of MTX-treated rats. The cytotoxicity caused by MTX + metabolites was higher than that observed in the H9c2 cells incubated with non-metabolized MTX group. The co-incubation of MTX with CYP450 and CYP2E1 inhibitors partially prevented the cytotoxicity observed in the MTX groups incubated with H9c2 cells, highlighting that the metabolism of MTX is relevant for its undesirable effects. The naphtoquinoxaline metabolite is described in heart and liver in vivo, highlighting that this metabolite accumulates in these tissues. It was demonstrated that MTX P450-mediated metabolism contributed to MTX toxicity.

Keywords Mitoxantrone - Metabolism - Bioactivation LC/MS - S9 fraction - Cardiotoxicity

$\begin{array}{ll}\text { Abbreviations } \\ \text { DAD } & \text { Photodiode array } \\ \text { DAS } & \text { Diallyl sulfide } \\ \text { DMEM } & \text { Dulbecco's modified eagle's medium } \\ \text { DMSO } & \text { Dimethyl sulfoxide } \\ \text { ESI } & \text { Electrospray ionization interface } \\ \text { GSH } & \text { Reduced glutathione } \\ \text { LC } & \text { Liquid chromatography } \\ \text { MS } & \text { Mass spectrometry } \\ \text { MTT } & 3 \text {-(4,5-dimethylthiazol-2-yl)-2,5- } \\ & \text { diphenyltetrazolium bromide } \\ \text { MTP } & \text { Metyrapone } \\ \text { MTX } & \text { Mitoxantrone } \\ \beta-N A D P H & \beta \text {-Nicotinamide adenine dinucleotide } \\ & \text { phosphate }\end{array}$




\section{Introduction}

Mitoxantrone (MTX) is an anticancer drug synthesized in the end of 1970s as an alternative to anthracyclines therapy (Canal et al. 1993). It has been largely used in the treatment of solid tumors, acute leukemia, lymphoma, prostate, and breast cancer (Seiter 2005) and, more recently, in the active forms of relapsing-remitting or secondary progressive multiple sclerosis (Neuhaus et al. 2006). Despite its broad utilization, MTX is potentially cardiotoxic (Seiter 2005; Avasarala et al. 2003).

The pharmacological activity of MTX is based on the ability to intercalate its planar electron-rich chromophore group in the DNA and to allow electrostatic interactions of its side chains with the phosphate moiety of DNA (Ehninger et al. 1990). Furthermore, MTX causes single and double breaks in DNA by stabilization of a complex formed between DNA and topoisomerase II (Ehninger et al. 1990; Seiter 2005). As a consequence, MTX inhibits DNA replication, RNA transcription, and also affects the cell cycle at various stages (Khan et al. 2010; Ehninger et al. 1990).

The oxidoreductive metabolism of MTX has a significant role on its antitumor effects. MTX has particular effectiveness in tumors with high contents of peroxidases (Brück and Brück 2011; Blanz et al. 1991), and it was verified that the inhibitory effect of MTX on cell growth was prevented by inhibiting the activity of cytochrome P450 mixed oxidase function in a human hepatoma-derived cell line (Duthie and Grant 1989). Similar results were obtained with a rat hepatocytes model (Mewes et al. 1993) and with human breast cancer cells (Li et al. 1995). Phorbol ester-stimulated human neutrophils can bioactive MTX through myeloperoxidase metabolism, and the generated metabolites can form adducts with DNA (Panousis et al. 1997). Furthermore, it was already demonstrated that a synthetic cyclic metabolite of MTX, the 8,11-dihydroxy-4(2-hydroxyethyl)-6-[[2-[(2-hydroxyethyl)amino]ethyl] amino]-1,2,3,4,7,12-hexahydronaphto-[2-3]-quinoxaline-712-dione or naphtoquinoxaline metabolite, causes cellular damage in neonatal cardiomyocytes isolated from rats (Shipp et al. 1993). This metabolite was already described as an in vivo MTX biotransformation product in humans, pigs, and rats (Blanz et al. 1991). With the exception of the study mentioned above (Shipp et al. 1993), to the best of our knowledge, there are no studies relating the bioproducts of MTX with its most serious undesirable effect: the late irreversible cardiotoxicity (Avasarala et al. 2003).

Pharmacokinetic studies showed that after intravenous administration, MTX has a rapid distribution followed by a slow elimination phase characterized by an extensive accumulation in highly perfused organs in humans and laboratory animals (Ehninger et al. 1990; Batra et al. 1986).
The metabolism of MTX involves the oxidation through the microsomal system and/or peroxidase enzymes such as neutrophil myeloperoxidase (Duthie and Grant 1989; Panousis et al. 1997). Moreover, the phase II metabolism has a relevant role in the MTX detoxification process, namely the conjugation with reduced glutathione (GSH) and glucuronic acid (Ehninger et al. 1990). Considering the interspecies variability, the main metabolic difference between rats and humans is that mono and dicarboxylic acid derivatives of MTX are major bioproducts of human metabolism, while in rats, they are residual (Blanz et al. 1991; Richard et al. 1991).

The difficulty to reproduce the oxidoreductive metabolism of MTX in in vitro conditions is extensively reported (Wolf et al. 1986; Richard et al. 1991; Basra et al. 1985; Kostrzewa-Nowak et al. 2007; Fisher and Patterson 1992; Fisher et al. 1993). Because of that, some authors use exogenously added isolated enzymes such as NADPH cytochrome P450 reductase (Kostrzewa-Nowak et al. 2007; Novak and Kharasch 1985) and peroxidase enzymes system (Blanz et al. 1991; Brück and Brück 2011) in order to circumvent this limitation. Nevertheless, from all in vitro methods, these are the less realistic ones. There are also metabolic studies employing more representative systems, namely using primary cultures of hepatocytes isolated from rats, rabbits, and humans (Richard et al. 1991), microsomes, and cytosol fractions isolated from rat livers (Wolf et al. 1986). The referred studies use HPLC/UV methods to detect and quantify the metabolites (Richard et al. 1991; Wolf et al. 1986), which limit the analysis due to the low sensitivity of UV detector and do not allow the structural elucidation of the metabolites. Alternative methods use liquid scintillation counting equipped with a radioactive flow detector (Richard et al. 1991), which is not commonly available in the majority of analytical laboratories and requires the undesirable use of labeled compounds.

We present herein a study that aims to assess the contribution of MTX metabolism to its cardiotoxicity. The metabolic profile of MTX was studied using the hepatic S9 fraction from phenobarbital-induced animals, which are considered a representative hepatic model for this purpose as it contains both phase I and II enzymes involved in the MTX metabolism (Brandon et al. 2003; Jia and Liu 2007). The MTX metabolites were separated by liquid chromatography (LC) coupled with a UV-VIS photodiode array detector (DAD) and accurately identified by electrospray ionization interface (ESI) mass spectrometry detector (MS) that possess a higher sensitivity and accuracy. The cytotoxic effects of MTX metabolites and the influence of CYP450 and CYP2E1 metabolism in the cytotoxicity of MTX were evaluated in the $\mathrm{H} 9 \mathrm{c} 2$ cells, a valuable cardiomyoblast in vitro model (Zordoky and El-Kadi 2007). An in vivo study was also performed in order to investigate 
whether the MTX metabolites previously found in vitro were also present in the liver and heart of rats after MTX intraperitoneal administration.

\section{Materials and methods}

\section{Chemicals}

All chemicals and reagents were of analytical grade. MTX hydrochloride, GSH, reduced $\beta$-nicotinamide adenine dinucleotide phosphate ( $\beta$-NADPH), metyrapone (MTP), diallyl sulfide (DAS), and 3-(4,5-dimethylthiazol-2-yl)-2,5diphenyltetrazolium bromide (MTT) were obtained from Sigma-Aldrich (St. Louis, MO, USA). Methanol, dimethyl sulfoxide (DMSO), and formic acid were obtained from Merck (Darmstadt, Germany).

Dulbecco's modified eagle's medium (DMEM) with $4,500 \mathrm{mg} / \mathrm{L}$ glucose and GlutMAX ${ }^{\mathrm{TM}}$, fetal bovine serum FBS, trypsin $(0.25 \%)$-EDTA $(1 \mathrm{mM})$, and antibiotic $(10,000 \mathrm{U} / \mathrm{mL}$ penicillin, $10,000 \mathrm{~g} / \mathrm{mL}$ streptomycin) were obtained from Gibco Laboratories (Lenexa, KS, USA).

\section{Animals}

Adult male Wistar rats (Charles River Laboratories, Barcelona, Spain) weighing 300-350 g were used. The animals were acclimated, housed in cages, with a temperature- and humidity-controlled environment. Food and water were provided ad libitum, and animals were subjected to a 12-h light-dark cycle. Animal experiments were licensed by Portuguese General Directory of Veterinary Medicine. Housing and experimental treatment of the animals were in accordance with the Guide for the Care and Use of Laboratory Animals from the Institute for Laboratory Research. The experiments complied with current Portuguese laws.

\section{Isolation of the hepatic S9 fraction}

The animals used for the hepatic S9 fraction isolation received phenobarbital $0.2 \%$ in drinking water for 1 week prior to the excision of the livers, in order to induce metabolism (Wolf et al. 1986). All the animals were monitored, and no signals of toxicity were observed.

The animals were killed through cervical dislocation. The livers were excised, washed with $100 \mathrm{mM}$ phosphate buffer, pH 7.4 (in order to remove the excess of blood), dried, and weighed. Livers were homogenized in $100 \mathrm{mM}$ phosphate buffer, pH 7.4 ( $1 \mathrm{~g} / 4 \mathrm{~mL}$ of buffer), and centrifuged $9,000 \times g, 20 \mathrm{~min}, 4{ }^{\circ} \mathrm{C}$. The supernatant contains the $\mathrm{S} 9$ fraction. All steps were carefully performed on ice.
An aliquot was used to quantify the protein levels by Lowry method (Rossato et al. 2011, Lowry et al. 1951).

Evaluation of the metabolic profile of MTX

Prior to the incubations, the protein density of the $\mathrm{S} 9$ fraction was adjusted to contain $4 \mathrm{mg} / \mathrm{mL}$ in $100 \mathrm{mM}$ phosphate buffer, pH 7.4 (Wolf et al. 1986). Samples were supplemented with $1 \mathrm{mM} \mathrm{NADPH}$ and $4 \mathrm{mM} \mathrm{GSH}$, as previously reported (Wolf et al. 1986). The incubations were carried out using $100 \mu \mathrm{M}$ MTX, at $37{ }^{\circ} \mathrm{C}$, for 4 -h. Aliquots of this mix were taken at the incubation times 0 and 4-h. One blank tube containing control supplemented S9 fraction (without MTX) was also incubated and further analyzed in order to evaluate possible matrix interferences. One tube containing only $100 \mu \mathrm{M}$ MTX $+1 \mathrm{mM}$ NADPH $+4 \mathrm{mM}$ GSH (without the $\mathrm{S} 9$ fraction) was also evaluated in order to rule out the possibility of artifacts formation.

At 0 and 4-h, methanol was added to the samples (ratio of 1:4 methanol) in order to precipitate the proteins and to extract MTX and the metabolites. Samples were centrifuged at $16,000 \times g, 5 \mathrm{~min}, 4^{\circ} \mathrm{C}$, and the supernatant was dried under nitrogen flux. The residues were re-suspended in $200 \mu \mathrm{L}$ of methanol and analyzed by LC/DAD-ESI/MS or in $100 \mu \mathrm{L}$ phosphate saline buffer for the assessment of cytotoxicity (in detail addressed below).

The incubations with S9 fraction were independently performed in 3 different days with $\mathrm{S} 9$ fraction isolated from 3 different animals.

In vivo metabolic study

A study using male Wistar rats was performed in order to verify the presence of MTX metabolites in liver and heart. Three animals received a single dose of $7.5 \mathrm{mg} / \mathrm{kg}$ of MTX, via intraperitoneal, and were killed 24-h after treatment. The animals were anesthetized with xilasin/ ketamine $(10 \mathrm{mg} / \mathrm{kg} / 100 \mathrm{mg} / \mathrm{kg})$ and were killed after diaphragm rupture. The livers and hearts were excised and processed as previously described (An and Morris 2010), with minors adaptations. Briefly, the organs were homogenized with $0.1 \mathrm{M}$ citric buffer containing $100 \mathrm{mg} / \mathrm{mL}$ ascorbic acid, $\mathrm{pH}$ 3.0. MTX, and its metabolites were extracted after the sequential addition of sulfosalicylic acid $(50 \mu \mathrm{L}$, final concentration of $5 \%)$ and acetonitrile $(150 \mu \mathrm{L})$ to the tissue homogenate $(200 \mu \mathrm{L})$. Samples were centrifuged at $16,000 \times g, 10 \mathrm{~min}, 4{ }^{\circ} \mathrm{C}$, and the supernatant was dried under nitrogen flux. The residues were re-suspended in $200 \mu \mathrm{L}$ methanol and analyzed by LC/ DAD-ESI/MS, as described below. Control organs (from animals that did not receive MTX) were also evaluated in order to assess matrix interferences. 


\section{LC-DAD/ESI-MS analysis}

A Finnigan Surveyor series LC, equipped with Lichrocart Purospher ${ }^{\circledR}$ Star reversed-phase column $(25 \mathrm{~cm} \times 4.6 \mathrm{~mm}$ inner diameter, $5 \mu \mathrm{M}, \mathrm{C} 18)$, was used. The samples were analyzed using aqueous $0.5 \%(\mathrm{v} / \mathrm{v})$ formic acid as solvent A and acetonitrile as solvent B (Brück and Brück 2011). The $\mathrm{pH}$ of the mobile phase was set to 3.0 with formic acid. The gradient profile was $90 \%(\mathrm{v} / \mathrm{v}) \mathrm{A} / 10 \%(\mathrm{v} / \mathrm{v}) \mathrm{B}$ for $1 \mathrm{~min}$ and, subsequently, the percentage of acetonitrile was ramped linearly to $80 \% \mathrm{~B}$ over a time span of $20 \mathrm{~min}$ (Brück and Brück 2011). The analysis was done at a flow rate of $0.5 \mathrm{~mL} / \mathrm{min}$. The sample injection volume was $25 \mu \mathrm{L}$. The chromatographic column was stabilized with the initial conditions for $10 \mathrm{~min}$. Double-online detection was done by a DAD and MS detectors. The MS detector was a Finnigan LCQ DECA XP MAX (Finnigan Corp., San Jose, CA, USA) quadrupole ion trap equipped with atmospheric pressure ionization source, using ESI. The vaporizer and the capillary voltages were $5 \mathrm{kV}$ and $4 \mathrm{~V}$, respectively. The capillary temperature was set at $325{ }^{\circ} \mathrm{C}$. Nitrogen was used as both sheath and auxiliary gas at flow rates of 90 and 35 , respectively (in arbitrary units). Spectra were recorded in positive ion mode between $\mathrm{m} / z, 250$ and 1,100.

Comparison of the cytotoxic effects of non-metabolized MTX and MTX metabolites in H9c2 cells

The H9c2 cell line was a generous gift from Dr. Vilma Sardão, Center for Neurosciences and Cellular Biology, University of Coimbra, Portugal. Cells were cultured in DMEM supplemented with $10 \%$ fetal bovine serum, $100 \mathrm{U} / \mathrm{mL}$ of penicillin, and $100 \mathrm{U} / \mathrm{mL}$ of streptomycin in $75 \mathrm{~cm}^{2}$ tissue culture flasks at $37{ }^{\circ} \mathrm{C}$ in a humidified atmosphere of $5 \% \mathrm{CO}_{2}, 95 \%$ air atmosphere. Cells were fed every 2-3 days and sub-cultured once they reached 70-80 \% confluence (Sardão et al. 2009a, b).

Cells were seeded at a density of 35,000 cells $/ \mathrm{mL}$ in 48-well plates (final volume of $250 \mu \mathrm{L}$; about 8,000 cells/ $\mathrm{cm}^{2}$ ) and were allowed to grow for 2 days. On the day of experiment, the medium was replaced and cells were divided into four groups (six-well per group): (a) control group (without treatment), (b) matrix group (the previously 4-h incubated S9 liver fraction enriched with $1 \mathrm{mM}$ NADPH and $4 \mathrm{mM} \mathrm{GSH}$ ), (c) MTX + metabolites group (the product of 4-h incubation of S9 liver fraction $+1 \mathrm{mM}$ $\mathrm{NADPH}+4 \mathrm{mM}$ GSH $+100 \mu \mathrm{M}$ MTX), and (d) nonmetabolized MTX group (S9 liver fraction $+1 \mathrm{mM}$ $\mathrm{NADPH}+4 \mathrm{mM}$ GSH $+100 \mu \mathrm{M}$ MTX, on time 0 of incubation). The presence/absence of metabolites in the groups (c) and (d), respectively, was confirmed by LC/ DAD-ESI/MS prior to the incubations. The cytotoxic effects were assessed after $24-\mathrm{h}$ incubation period at $37{ }^{\circ} \mathrm{C}$ through the reduction in MTT assay (described in more detail below). Three independent experiments were performed (with cells seeded on different days and incubated with samples obtained from S9 fraction isolated from 3 different animals).

Assessment of the protective effect of the co-incubation of MTX and P450 cytochrome metabolism inhibitors in $\mathrm{H} 9 \mathrm{c} 2$ cells

The CYP450 inhibitor MTP (0.5 mM) (Duthie and Grant 1989; Mewes et al. 1993; $\mathrm{Li}$ et al. 1995) and the CYP2E1 subtype inhibitor DAS $(150 \mu \mathrm{M})$ (Pontes et al. 2010) were co-incubated with MTX $(100 \mathrm{nM}$ and $1 \mu \mathrm{M})$ at $37^{\circ} \mathrm{C}$ for 96-h. MTP was dissolved in DMSO; thus, a group with only the vehicle (DMSO $0.1 \%$ ) was also included. The potential protective effect of metabolism inhibitors in the cytotoxicity of MTX was assessed through the MTT assay.

\section{MTT reduction assay}

The cytotoxicity upon H9c2 cells was assessed through the reduction of MTT. At the end of incubation period, the medium was removed and the cells were washed with phosphate saline buffer, $\mathrm{pH} 7.4$, followed by the addition of fresh cell culture medium containing $0.5 \mathrm{mg} / \mathrm{L}$ MTT and incubated at $37{ }^{\circ} \mathrm{C}$ in a humidified, $5 \% \mathrm{CO}_{2}, 95 \%$ air atmosphere for 4-h. After this incubation period, the cell culture medium was removed and the formed formazan crystals were dissolved in $100 \%$ DMSO. The absorbance was measured at $550 \mathrm{~nm}$ in a multi-well plate reader (BioTek Instruments, Vermont, US). The percent of the MTT reduction relative to control cells was used as the cytotoxicity measure (Silva et al. 2011).

Statistical analysis

Results are presented as mean \pm standard deviation ( $n=18$ per condition out of 3 independent studies). Since the MTT results passed the normality test, a parametric test was used. One-way ANOVA was performed to compare means of different groups followed by the Student-Newman-Keuls post hoc test. Statistical significance was accepted at $p$ values $<0.05$.

\section{Results}

Metabolic profile of MTX after incubation with supplemented rat hepatic S9 fraction

It was possible to demonstrate the metabolism of MTX using hepatic S9 fraction isolated from adult rats 
supplemented with $1 \mathrm{mM}$ NADPH and $4 \mathrm{mM}$ GSH. As shown in the Fig. 1, the MTX has a retention time of about 15 min. After 4-h incubation of the MTX with the supplemented S9 fraction, a significant decrease of about $35 \%$ in the MTX peak area was observed, highlighting MTX metabolism in this in vitro system. Moreover, after 4-h, 5 additional chromatographic peaks were present and were numerically identified on the LC-DAD/ESI-MS chromatogram (Fig. 1). The peaks 1 and 3 were present in small amounts in time 0 , their areas increasing at time $4-h$. The peaks 2, 4 (trace), and 5 were only found after the 4-h incubation period (Fig. 1). In Fig. 2, the UV-VIS DAD spectral properties of MTX and its metabolites are presented, showing that all metabolites identified still possess the tricyclic planar chromophore group.

Data about the retention times, the MS fragmentation pattern, and the proposed structure of MTX and its metabolites are summarized on Table 1. MTX was unequivocally identified on the basis of its already described UV-VIS spectral features (Fig. 2) (Ehninger et al. 1990) and its parent molecular ion mass $\left([\mathrm{M}+\mathrm{H}]^{+}\right.$) at $m / z 445$ (Brück and Brück 2011). The metabolite 5, the naphtoquinoxaline, was also already reported (Blanz et al. 1991; Brück and Brück 2011), and it has UV-VIS spectrum with maximum absorbance at 583 and $634 \mathrm{~nm}$ (Fig. 2). Its parent molecular ion mass $\left([\mathrm{M}+\mathrm{H}]^{+}\right)$is $m / z$ 443. Both MTX and metabolite 5 have the same MS/MS daughter fragmentation derived from cleavages on the side chain: $m / z 358$ for MTX and $m / z \quad 356$ for metabolite $5\left(\left[\mathrm{M}+\mathrm{H}^{+}\right]-\mathrm{CH}_{2}=\right.$ $\mathrm{CHNH}_{2} \mathrm{CH}_{2} \mathrm{CH}_{2} \mathrm{OH}$ ), and $m / z, 384$ for MTX and $m / z, 382$ for metabolite $5\left(\left[\mathrm{M}+\mathrm{H}^{+}\right]-\mathrm{NH}_{2} \mathrm{CH}_{2} \mathrm{CH}_{2} \mathrm{OH}\right]$.
The metabolites 1 and 2 are probably the MTX conjugates with one and two molecules of GSH, resulting in the parent molecular ion mass $\left([\mathrm{M}+\mathrm{H}]^{+}\right)$at $\mathrm{m} / z \quad 750$ (metabolite 1) and $\left([\mathrm{M}+\mathrm{H}]^{+}\right.$) at $m / z$ 1,055 (metabolite 2) (Table 1), the same parent molecular ion previously described for these metabolites (Blanz et al. 1991). Regarding metabolite 1 , the same pattern of fragmentation of parent ion 750 was obtained $(\mathrm{m} / \mathrm{z}=477$ and $\mathrm{m} / \mathrm{z}=621)$ (Supplementary data). However, considering the metabolite 2, the fragmentation of the ion at $\mathrm{m} / \mathrm{z}, 1,055$ results in fragments $(\mathrm{m} / \mathrm{z} 845$ and 775) different from those found in the study performed by Blanz and co-workers $(\mathrm{m} / \mathrm{z} 926$ and 797) (Table 1). Thus, the occurrence of structural rearrangements or the insertion of the GSH molecules in other positions is possible. Metabolite 1 presents maximum absorbance peaks at 619 and $673 \mathrm{~nm}$, and metabolite 2 presents maximum absorbance peaks at 613 and $661 \mathrm{~nm}$ (Fig. 2). The LC/DAD-ESI/MS analysis does not allow a conclusion about the site of GSH conjugation. However, we propose the GSH adduct on the dihydroxybenzene part of MTX molecule for metabolite 1 based on the previous work of Mewes and co-workers, which elucidated GSH conjugates chemical structure through two-dimensional ${ }^{1} \mathrm{H}-{ }^{13} \mathrm{C}$-heteronuclear multiple-bond connectivity NMR technique (Mewes et al. 1993).

The metabolite 3 presents an UV-VIS spectrum similar to $\operatorname{MTX}$ (Fig. 2). Its mass spectrum (Table 1) showed a parent molecular ion mass $\left([\mathrm{M}+\mathrm{H}]^{+}\right)$at $m / z 503$ and MS/ MS fragmentations with $m / z \quad 442\left([\mathrm{M}+\mathrm{H}]^{+}-\mathrm{NH}_{2} \mathrm{CH}_{2}\right.$ $\left.\mathrm{CH}_{2} \mathrm{OH}\right)$ and $416\left([\mathrm{M}+\mathrm{H}]^{+}-\mathrm{CH}_{2}=\mathrm{CHNH}_{2} \mathrm{CH}_{2} \mathrm{CH}_{2} \mathrm{OH}\right)$. The parent ion suggests that this compound has a
Fig. 1 Overlap of LC/DADESI/MS chromatograms of time 0 - and 4-h incubation of rat hepatic $\mathrm{S} 9$ fraction $(4 \mathrm{mg} / \mathrm{mL})$ with $100 \mu \mathrm{M}$ MTX $+1 \mathrm{mM}$ $\mathrm{NADPH}+4 \mathrm{mM} \mathrm{GSH}$

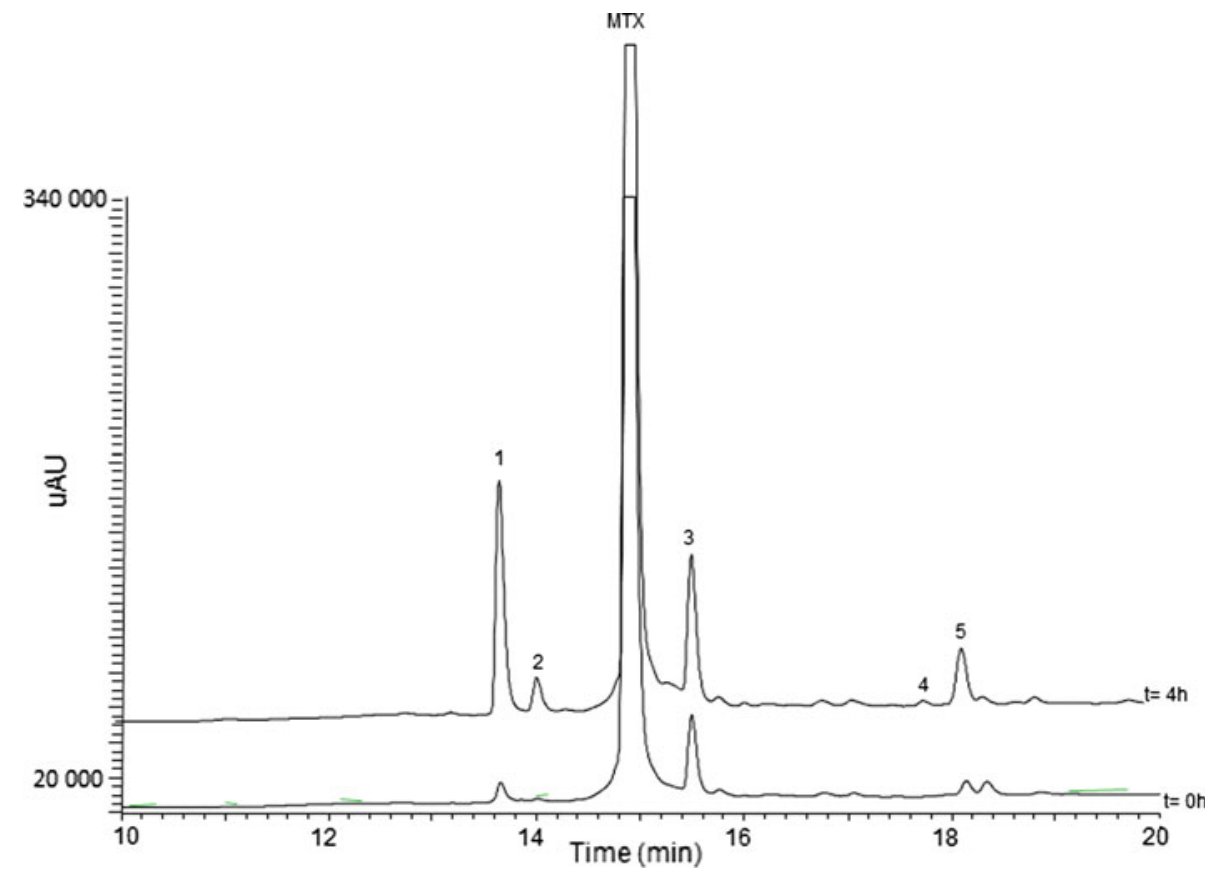




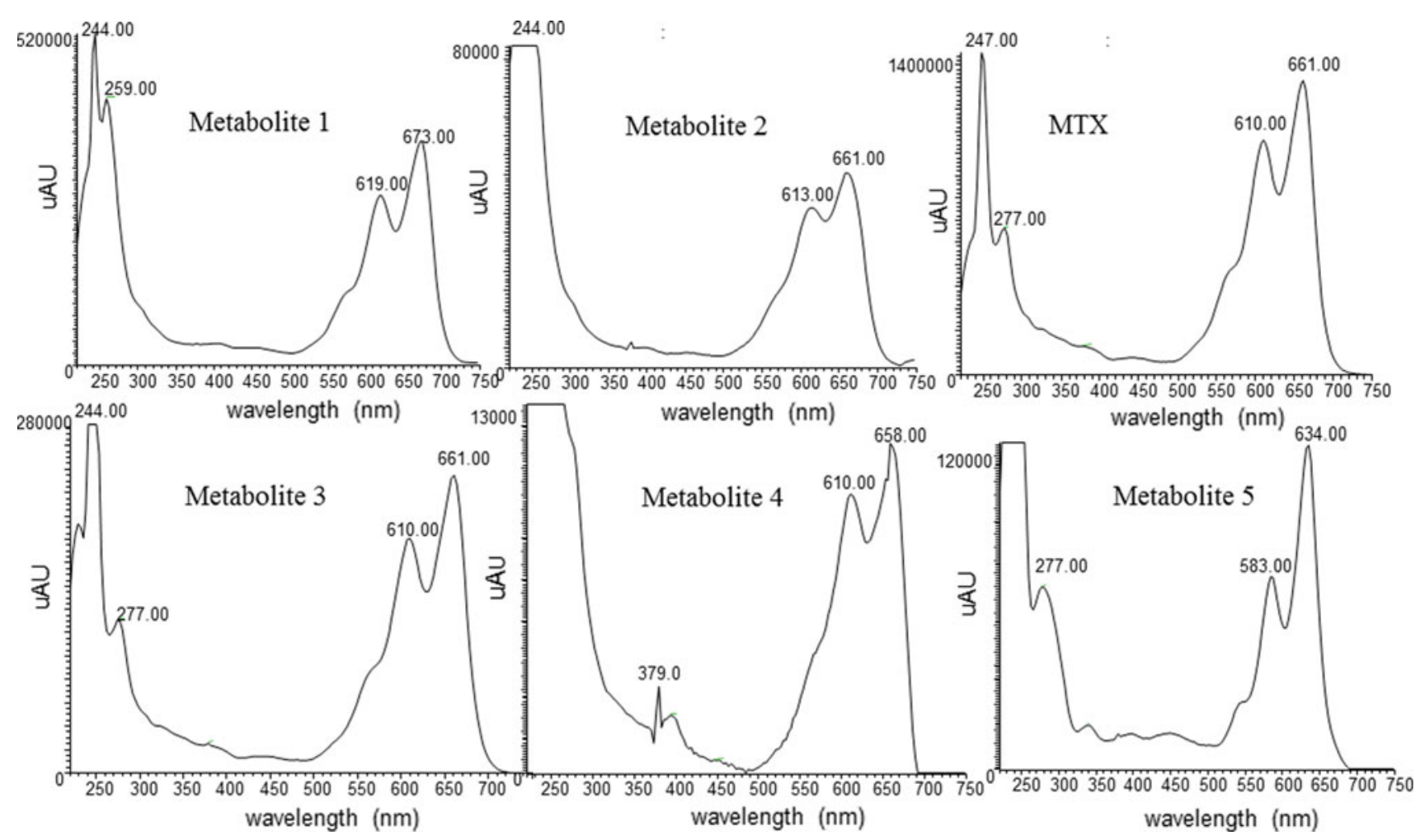

Fig. 2 UV-VIS spectral data (between 240 and $700 \mathrm{~nm}$ ) of MTX and its metabolites after 4-h incubation with rat hepatic S9 fraction supplemented with $1 \mathrm{mM}$ NADPH $+4 \mathrm{mM} \mathrm{GSH}$. The metabolites

$\mathrm{CH}_{3} \mathrm{COO}$ - group introduced in the original molecule of MTX (Table 1).

Metabolite 4 was found as a trace (Fig. 1), and it presents maximum absorbance at 610 and $658 \mathrm{~nm}$ (Fig. 2). Due to the small amount of this compound, the only information given by the mass spectrum was the parent ion $\left([\mathrm{M}+\mathrm{H}]^{+}\right)$at $m / z$ 455. Based on these data, we proposed for metabolite 4 the chemical structure presented on Table 1. A compound with the same molecular ion mass was previously described and associated with the oxidative metabolism of MTX (Brück and Brück 2011).

The analysis of the blank sample (containing only the supplemented S9 fraction, without the addition of MTX) revealed no relevant interferences from the matrix on the retention times of interest. Similarly, no peaks were observed at the same retention times in the sample containing only $100 \mu \mathrm{M}$ MTX $+1 \mathrm{mM}$ NADPH $+4 \mathrm{mM}$ GSH (without the S9 fraction) (data not shown).

Metabolic profile of liver and heart extracts of MTX-treated male rats

The metabolic profile was determined in vivo through the evaluation of liver and heart extracts of rats that received $7.5 \mathrm{mg} / \mathrm{kg}$ of MTX 24-h before. MTX and its metabolites were extracted and concentrated as described in the number designations are in accordance with the chromatogram showed in Fig. 1

"Materials and methods" section and analyzed through LC/DAD-ESI/MS. Control liver and heart extracts were also analyzed in order to determine matrix interferences. More attention was given in the search and identification of the metabolite 5 due to its known pharmacological activity (Brück and Brück 2011; Shipp et al. 1993).

In the liver extracts of the animals treated with MTX (Fig. 3a), it was possible to observe the presence of MTX, with a retention time of about $15 \mathrm{~min}$, and 7 additional peaks when compared to control livers (data not shown). All the compounds identified in the chromatogram as metabolites of MTX are compounds that absorb radiation between 500 and $700 \mathrm{~nm}$. At the retention time of $18.16 \mathrm{~min}$, it was identified the metabolite 5 due to its UVVIS spectrum characteristics and the MS fragmentation (data not shown). Further structural identification of the other metabolites was not possible due to the huge background presented in the analysis. The metabolites that have the retention times of 13.21 and 13.70 min possess a UVVIS spectrum profile similar to the metabolite 1 , with maximum absorbance in the visible region set at 619 and $673 \mathrm{~nm}$ (data not shown). The metabolite with the retention time of $14.04 \mathrm{~min}$ has the same absorbance peaks in the visible region of the metabolite 4, namely 610 and $658 \mathrm{~nm}$ (data not shown). The metabolites with the retention times of $14.62,15.39$, and 15.79 min have a UV-VIS spectrum 
Table 1 Proposed chemical structure, retention times, and mass spectrum of MTX and its metabolites obtained through LC-DAD/ESI-MS after 4-h incubation of $100 \mu \mathrm{M}$ MTX in supplemented S9 fraction

\begin{tabular}{|c|c|c|c|c|}
\hline Compound & Chemical structure & Retention time (LC/MS) (min) & MS parent-ion mass $[\mathrm{m} / \mathrm{z}]$ & MS/MS daughter ions $[\mathrm{m} / \mathrm{z}]$ \\
\hline MTX & & 15.01 & 445 & $384+358$ \\
\hline 1 & & 13.74 & 750 & $621+477$ \\
\hline 2 & & 14.10 & 1,055 & $845+775$ \\
\hline 3 & $\Gamma$ & 15.61 & 503 & $442+416$ \\
\hline 4 & & 17.86 & 455 & - \\
\hline 5 & & 18.28 & 443 & $382+356$ \\
\hline
\end{tabular}

with absorbance set at 610 and $661 \mathrm{~nm}$, which are the absorbance maximum peaks of metabolite 3 and MTX.

In heart extracts (Fig. 3b), it was only observed the presence of MTX (retention time about $15 \mathrm{~min}$ ) and metabolite 5 (retention time about $18 \mathrm{~min}$ ), in trace amounts. Both compounds were identified through analysis of their DAD spectrum properties and MS fragmentation. None of the other peaks were identified as other MTX metabolites.

The metabolites of MTX increase the toxicity

in a cardiomyoblast in vitro model

The cytotoxicity of MTX and pre-incubated MTX metabolic S9 fraction was assessed in cardiomyoblast model through the reduction of MTT assay after a time period incubation of 24-h. The treatment groups result from the extracts of the previous incubations of the supplemented S9 fraction with $100 \mu \mathrm{M}$ MTX (with the exception of the matrix group, which did not contain MTX) at time 0 (non-metabolized MTX) or 4-h (MTX and its metabolites). The presence of MTX metabolites after 4-h incubation with $\mathrm{S} 9$ fraction was previously confirmed by LC/DAD-ESI/MS analysis, highlighting the presence of peaks of metabolites and a reduction of $35 \%$ of the MTX content at this time point (Fig. 1). Figure 4 presents the cytotoxicity results expressed as percent of the MTT reduction compared to control. As it can be seen, the incubation with the matrix group ( $\mathrm{S}$ 9 fraction $+1 \mathrm{mM}$ $\mathrm{NADPH}+4 \mathrm{mM}$ GSH after 4 -h incubation at $37{ }^{\circ} \mathrm{C}$ ) did not reveal any measurable toxic effect compared to control (96 $\pm 2 \%$ vs. $100 \pm 1 \%$, respectively). The MTX group and the MTX + metabolites group showed a significantly decrease in \% of MTT reduction compared to control $(79 \pm 2 \%$ for the MTX group and $67 \pm 2 \%$ for the MTX + metabolites group). In the group MTX + metabolites, the cytotoxic effects were significantly more pronounced, even with $35 \%$ less MTX, compared to MTX group, highlighting that its metabolism increases the cytotoxicity caused by MTX in the cardiomyoblast model. 
Fig. 3 LC/DAD-ESI/MS

chromatograms of (a) liver and

(b) heart extracts of MTX-

treated rats. Male Wistar rats received $7.5 \mathrm{mg} / \mathrm{kg}$ MTX, via intraperitoneal, and they were euthanized 24-h after treatment.

The livers and hearts extracts were obtained as described in the "Materials and methods" section

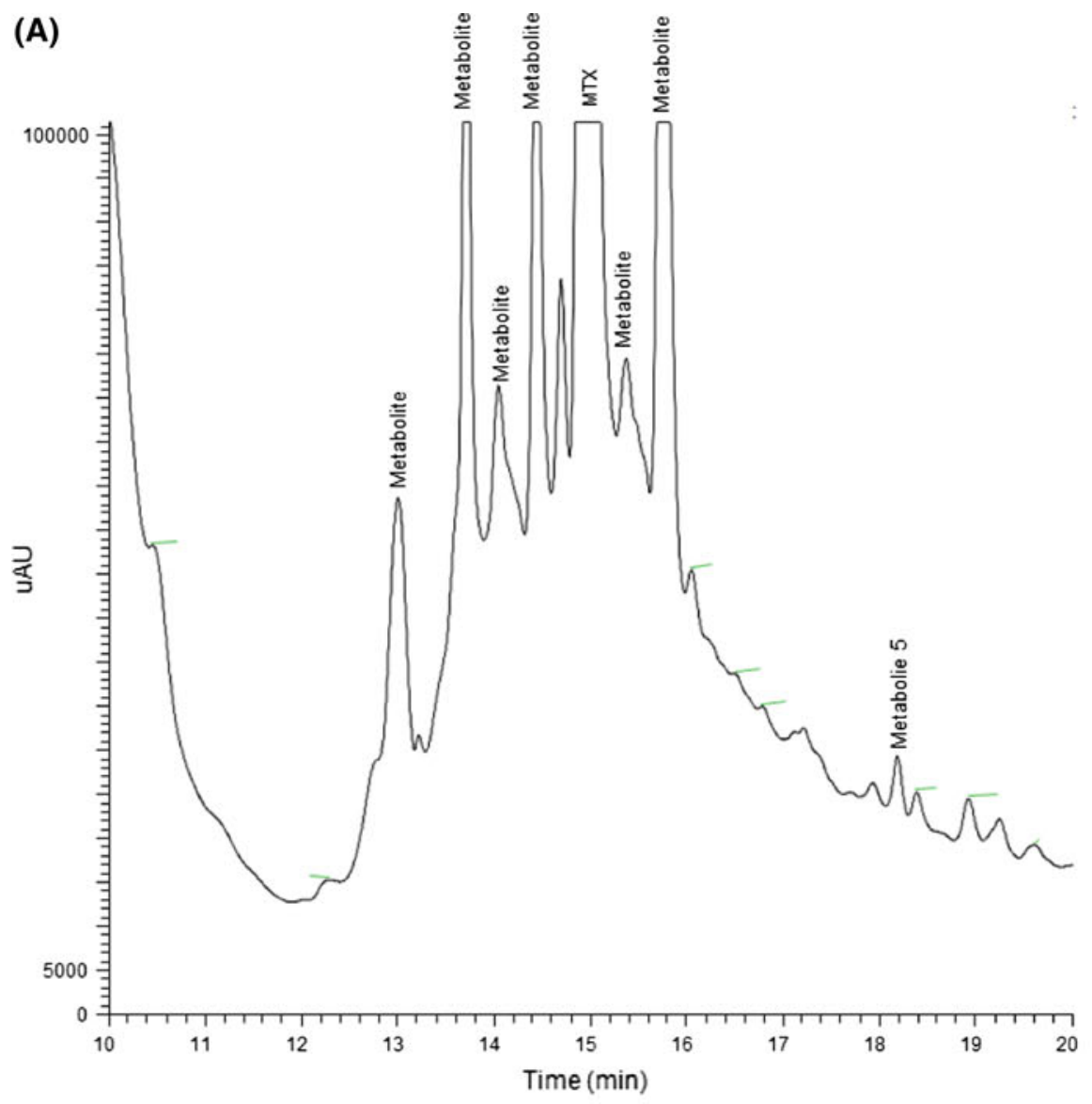

(B)

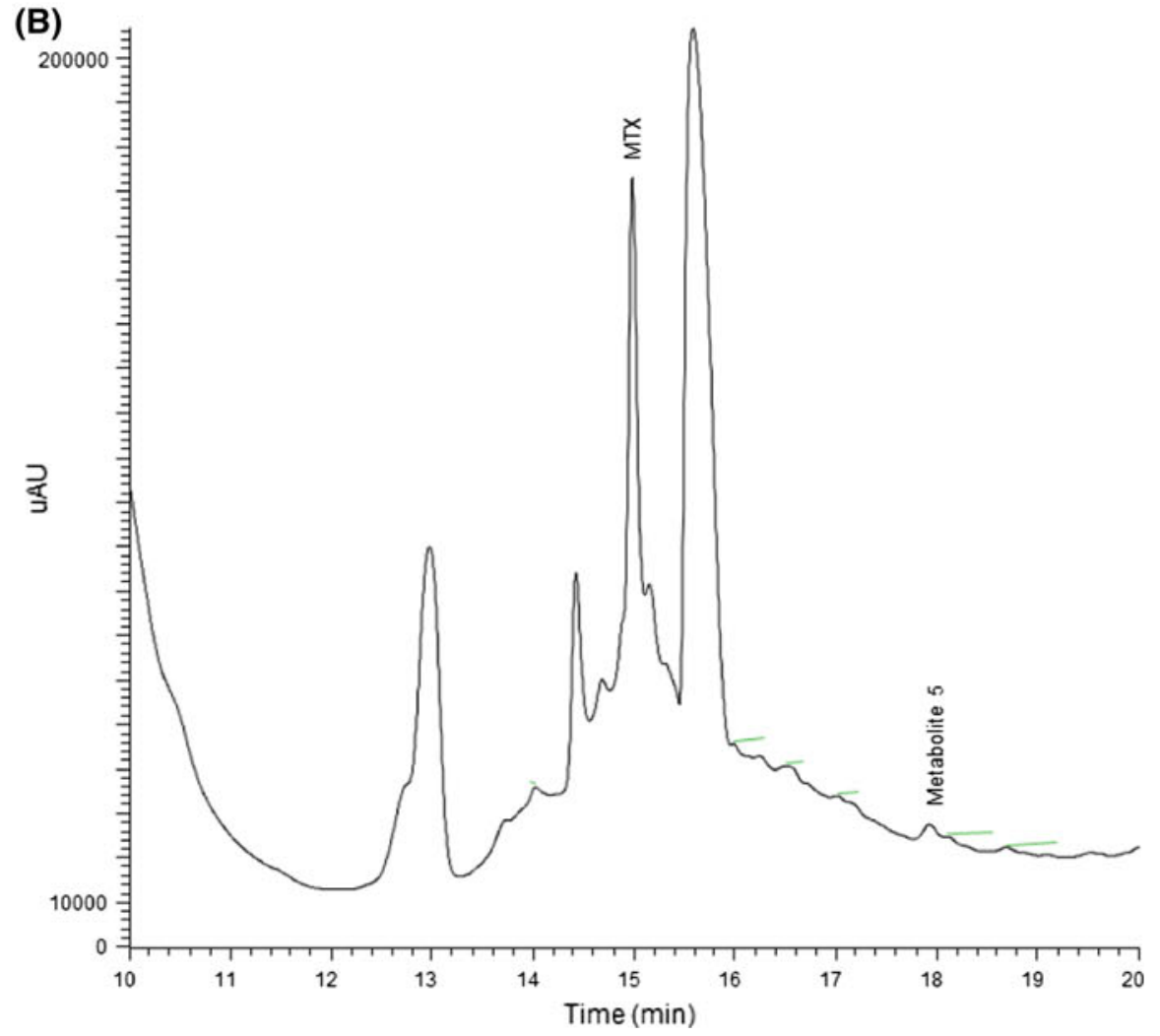


$24 \mathrm{~h}$

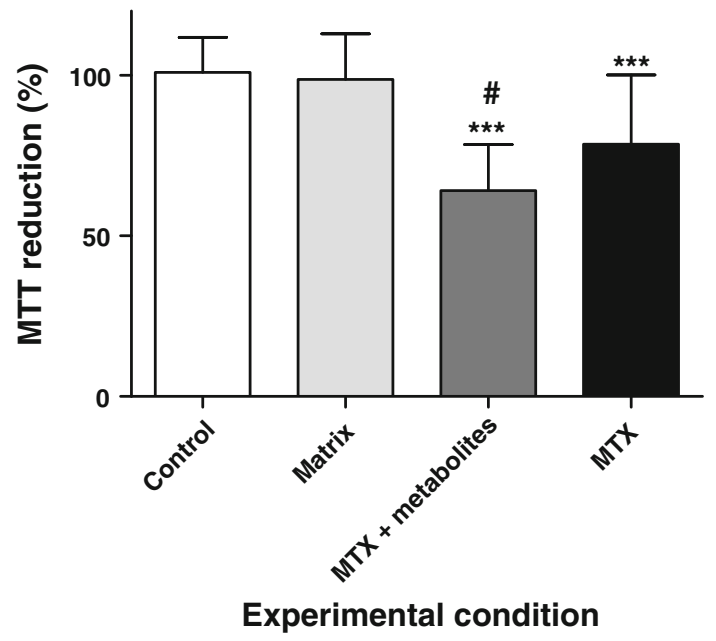

Fig. 4 Cytotoxic effects of MTX and MTX + metabolites in H9c2 cells after 24-h incubation. Results are presented as mean \pm standard deviation from 18 per condition out of 3 independent experiments. Statistical comparisons were made using One-way ANOVA followed by the Student-Newman-Keuls post hoc test $(* * * p<0.001$ vs. control; ${ }^{*} p<0.05$ vs. MTX group)

MTX cytotoxicity is prevented by the co-incubation of MTX with CYP450 and CYP2E1 inhibitors

Trying to counteract the cytotoxicity of MTX, the effects of CYP450 inhibitor MTP $(0.5 \mathrm{mM})$ and CYP2E1 inhibitor DAS $(150 \mu \mathrm{M})$ were evaluated through the MTT reduction assay. MTX (100 $\mathrm{nM}$ and $1 \mu \mathrm{M})$ was co-incubated with MTP or DAS at $37{ }^{\circ} \mathrm{C}$ for $96-\mathrm{h}$. Data are presented in Fig. 5.

In the presence of MTP, the cytotoxic effects observed after MTX incubation were significantly reduced compared to those observed with MTX alone: the \% of MTT reduction was $69 \pm 5 \%$ for the MTX $(100 \mathrm{nM})$ group versus $81 \pm 5 \%$ for the MTX $(100 \mathrm{nM})+$ MTP $(0.5 \mathrm{mM})$ group and $63 \pm 7 \%$ for the MTX $(1 \mu \mathrm{M})$ group versus $74 \pm 1 \%$ for the MTX $(1 \mu \mathrm{M})+\operatorname{MTP}(0.5 \mathrm{mM})$ group (Fig. 5a). The incubation with MTP alone or the DMSO $0.1 \%$ group did not produce any measurable toxic effect ( $99 \pm 5 \%$ and $95 \pm 2 \%$, respectively) compared to control $(100 \pm 4 \%)$.

A partial reversion of the cytotoxicity of MTX using the CYP2E1 subtype inhibitor DAS $(150 \mu \mathrm{M})$ was also observed. The co-incubation of MTX $(100 \mathrm{nM})+$ DAS $(150 \mu \mathrm{M})$ caused less toxic effects $(73 \pm 5 \%)$ compared to MTX $(100 \mathrm{nM})$ alone $(64 \pm 4 \%)$. The same was observed in the MTX $(1 \mu \mathrm{M})+$ DAS $(150 \mu \mathrm{M})(71 \pm 4)$ versus MTX $1 \mu \mathrm{M}$ group $(59 \pm 8)$. The $\%$ reduction in MTT in the DAS $(150 \mu \mathrm{M})$ group $(101 \pm 4 \%)$ was similar to those observed in the control group $(100 \pm 6 \%)$ (Fig. 5b).
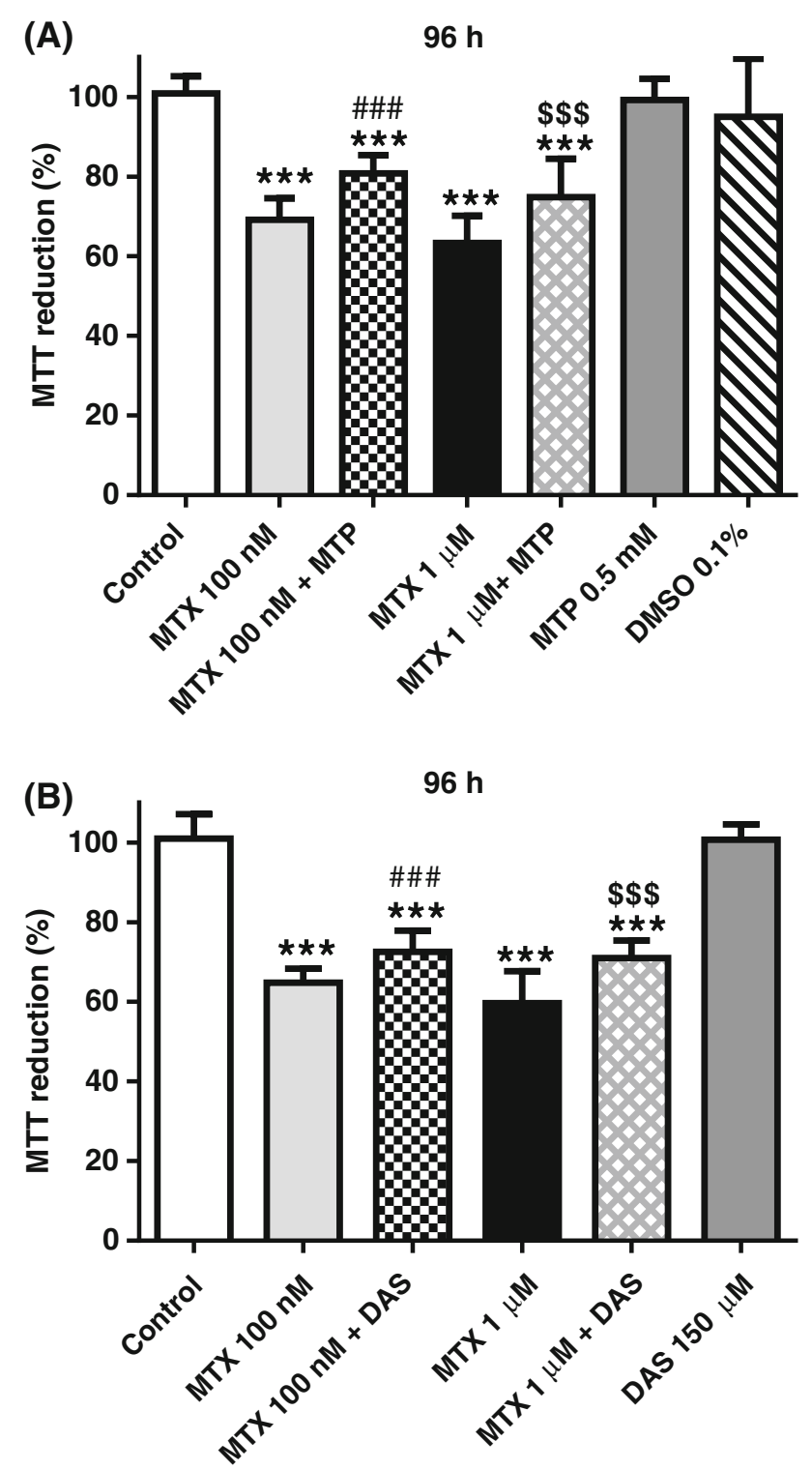

Fig. 5 Protective effects of (a) MTP $(0.5 \mathrm{mM})$ and (b) DAS $(150 \mu \mathrm{M})$ in the cytotoxic effects of MTX $(100 \mathrm{nM}$ and $1 \mu \mathrm{M})$ after 96-h co-incubation. Results are presented as means $(\%) \pm$ standard deviation ( $n=18$ per condition out of 3 independent experiments). Statistical comparisons were made using One-way ANOVA test followed by the Student-Newman-Keuls post hoc test $(* * * p<0.001$ vs. control; ${ }^{\# \#} p<0.001$ vs. $100 \mathrm{nM} ;{ }^{\$ \$ \$} p<0.001$ vs. $\left.1 \mu \mathrm{M}\right)$

\section{Discussion}

The major findings of the present work are as follows: (a) the description of the metabolic profile of MTX after incubation with the rat hepatic S9 fraction; (b) the finding that MTX and the naphtoquinoxaline metabolite are present in the extracts of hearts and livers of male Wistar rats treated with MTX 24-h before; (c) the demonstration of the increase in the MTX cytotoxicity in the presence of its metabolites toward $\mathrm{H} 9 \mathrm{c} 2$ cells; and (d) the partial 
prevention of the MTX cytotoxicity with the inhibition of the CYP450 and CYP2E1 metabolism in the H9c2 cells.

To the best of our knowledge, this is the first time that the metabolism of MTX through the supplemented rat hepatic S9 fraction is presented. Using this approach, 5 MTX metabolites were identified that keep the chromophore group (Fig. 2), including the naphtoquinoxaline metabolite (metabolite 5) and a novel metabolite that was never described before (an acetoxy ester derivative, metabolite 3) (Table 1). The maintenance of the chromophore group suggests that all these metabolites possibly maintain the ability to intercalate into DNA base pairs since the tricyclic planar structure is essential for MTX activity (Hsin et al. 2008).

In order to overpass the described difficulty to stimulate MTX metabolism in vitro (Duthie and Grant 1989; Richard et al. 1991; Kostrzewa-Nowak et al. 2007) animals received, as already reported, $0.2 \%$ phenobarbital in drinking water for 1 week prior to the $S 9$ fraction isolation to induce its metabolic systems (Wolf et al. 1986; Novak and Kharasch 1985). The liver S9 fractions are considered one of the most representative sub-cellular metabolic in vitro systems because these fractions contain phase I and phase II enzymes (Jia and Liu 2007; Yoshihara et al. 2001; Brandon et al. 2003) and are much more illustrative of a real situation than studies using exogenously and purified added enzymes (Jia and Liu 2007; Brandon et al. 2003). Subsequent analysis of S9 obtained extracts through LC/ DAD-ESI/MS presented in this work allowed an accurate separation and identification of the metabolic profile of MTX (Fig. 1; Table 1).

Studies discuss whether MTX suffers one-electron reduction or two-electron reduction (Wolf et al. 1986; Butler and Hoey 1987; Duthie and Grant 1989; Fisher and Patterson 1992). In fact, the reduction in MTX by flavin reductases is not facilitated due to its low one-electron reduction potential (Fisher and Patterson 1992). Hence, the preferential pathway of MTX metabolism is the two-electron reduction as evidenced by the prevention of the MTX bioactivation with the inhibition of cytochrome P450mediated metabolism (Li et al. 1995; Duthie and Grant 1989; Mewes et al. 1993) that was also observed in this work (Fig. 5).

The interest in the study of the metabolism of MTX has emerged due to the discover that the naphtoquinoxaline metabolite, which herein we called metabolite 5 (Table 1), is involved in the anti-tumoral effect of MTX (Feofanov et al. 1997; Mewes et al. 1993; Panousis et al. 1997). The metabolite 5 was already described as the product of MTX metabolism through heme containing enzymes systems, CYP450, and peroxidases (Brück and Brück 2011; Blanz et al. 1991). Other studies suggest that this metabolite certainly has a significant role in the pharmacological activity of MTX (Panousis et al. 1997; Feofanov et al. 1997; Mewes et al. 1993; Shipp et al. 1993) and may be associated with MTX-induced adverse effects since it causes ATP depletion in neonatal cardiomyocytes isolated from rats (Shipp et al. 1993).

The conjugation with GSH, resulting in the metabolites 1 and 2 (Table 1), is the main detoxifying pathway of the MTX (Mewes et al. 1993). These metabolites were previously found in studies with rat hepatic microsomes or cytosol incubated with $1 \mathrm{mM}$ MTX (Wolf et al. 1986) and after incubation of $60 \mu \mathrm{g} / \mathrm{mL}$ MTX with horseradish peroxidase supplemented with $25 \mathrm{mg}$ GSH (Blanz et al. 1991). To the best of our knowledge, the metabolite 3 (Table 1) was never associated with the MTX metabolism, although it is important to refer that it is the first time that a complex enzymatic system coupled to LC/DAD-ESI/MS analysis of MTX metabolites is presented. We suggest that the acetoxy derivative can result from an $\mathrm{N}$-oxygenation in the aromatic amine, followed by an acetylation. In fact, in primary and secondary aromatic amines, $N$-oxygenation by cytochrome P450 usually results in the formation of arylhydroxylamines, which can be converted by $N$-acetyltransferases (present in $\mathrm{S} 9$ fraction), functioning as $O$-acetyltransferases, to acetoxy esters (Parkinson and Ogilvie 2008).

As already stated, in our work, the metabolite 4 is present only in trace amounts (Fig. 1). Thus, it was not possible to obtain the complete MS fragmentation pattern. However, the MS parent-ion mass of this compound is set at $m / z$ 455. Recently, a study with horseradish, lacto, and lignin peroxidases incubated with MTX demonstrated the presence of a metabolite with the same parental ion of metabolite 4 (Brück and Brück 2011). Thus, based on this study, it was suggested that the metabolite 4 could have the proposed structure found on Table 1, the monocarboxylic acid derivative. Mono and dicarboxylic acid MTX derivatives were already associated with rat metabolism in very low and variable levels (Blanz et al. 1991; Richard et al. 1991), which is in accordance with the trace amounts found in our experiment with rat liver S9 fraction. In contrast, they are considered the main metabolites of MTX in rabbits and humans (Blanz et al. 1991; Richard et al. 1991), highlighting the interspecies differences in the metabolic profile of MTX.

An in vivo study was also performed to demonstrate the metabolic profile of MTX and determine the metabolites in the rat liver and heart (Fig. 3). The presence of MTX and the metabolite 5 in both organs was shown. Despite this metabolite has been already described in the urine of humans and many laboratory animals (Blanz et al. 1991), to the best of our knowledge, it is the first time that the presence of the naphtoquinoxaline metabolite is described in these organs in an in vivo situation. Furthermore, in liver extracts, 7 additional metabolites were found that preserve 
the chromophore group. It is known that MTX is rapidly and extensively distributed in tissues (An and Morris 2010), accumulating in organs such as liver and heart (Ehninger et al. 1990). In humans, MTX was detected 35 days after a single dose of $12 \mathrm{mg} / \mathrm{m}^{2}$ in liver $(1,140 \mathrm{ng} /$ $\mathrm{g}$ wet weight) and heart (716 ng/g wet weight) (Batra et al. 1986). The results presented here suggest that, as it happens with MTX, the naphtoquinoxaline metabolite also accumulates in hepatic and cardiac tissue.

In the second part of this work, the effects of the MTX bioactivation in a cardiomyoblast cellular in vitro model were studied. The cellular damage assessed through the MTT reduction assay was evaluated after 24-h incubation with the extracts of previous incubation of supplemented hepatic S9 fraction with $100 \mu \mathrm{M}$ MTX at time 0 or 4-h (containing about $35 \%$ less MTX than at time 0 and the metabolites presented in the Fig. 1; Table 1). As it can be seen in the Fig. 4, even with less amounts of MTX, the presence of the metabolites significantly increased the cytotoxicity when compared to the non-metabolized MTX group. A complex metabolic extract containing 5 in vitro obtained metabolites (including the metabolite 5) was used, and the results presented herein suggest the relevance of MTX metabolism for cardiac damage. The unique study correlating the bioactivation of MTX with its cardiotoxicity is in agreement with our results since it shows that the incubation of the previously synthesized and purified cyclic metabolite $(39 \mu \mathrm{M})$ (here named as metabolite 5, Table 1) in neonatal cardiomyocytes isolated from rats caused a partial depletion of the ATP content (Shipp et al. 1993). Thus, it seems that the bioactivation of MTX can be involved in both antitumoral and cardiotoxic effects. However, the mechanisms of pharmacological and adverse effects of MTX may differ since it was already demonstrated that the iron chelator ICRF-7 elicits cardioprotective effects in isolated neonatal rat cardiomyocytes and prevents the high-dose MTX lethality on CD-1 mice without counteracting the antitumor effect in two different tumor cell lines and in an in vivo model of leukemia (Shipp et al. 1993).

This is the first time that the prevention of MTX-induced cytotoxic effects by co-incubation with CYP450 metabolism inhibitors is evidenced in a cardiomyoblast model. As shown in the Fig. 5, the inhibition of CYP450-mediated metabolism significantly decreased the cytotoxicity observed with the same concentration of MTX in the absence of the broad CYP inhibitor MTP, highlighting that the in situ metabolism of MTX may also contribute to its toxicity. Another major finding of this work is that, for the first time, it is shown that similar protective results are obtained with the co-incubation with DAS, a CYP2E1 subtype inhibitor (Fig. 5b). The heart is an organ expressing both cytochrome P450 enzymes and NADPH cytochrome reductase (Duthie and Grant 1989; Yoshihara et al. 2001). CYP2E1 is markedly abundant in heart, and we showed here that it contributes to MTX cytotoxicity. The partial protection evidenced by the CYP inhibitors may be related to the direct toxic effect of MTX or the involvement of other enzymes present in the H9c2 cells that can promote the bioactivation of MTX. There are studies showing that the use of MTP prevents the cytotoxicity of MTX, namely in MCF7 human breast cancer cells ( $\mathrm{Li}$ et al. 1995), in human hepatoma-derived cells (Duthie and Grant 1989; Mewes et al. 1993), and rat hepatocytes (Mewes et al. 1993). Moreover, it was observed a proportional relationship between the cellular metabolic competences and the sensibility to MTX cellular damage, since rat hepatocytes are more susceptible to the cytotoxicity than hepatoma-derived cells (Mewes et al. 1993).

In summary, we presented the metabolic profile of MTX after incubation with rat hepatic S9 fraction. Five potentially MTX metabolites were presented, including the metabolite 3 that was never described before and the naphtoquinoxaline metabolite (metabolite 5). The metabolite 5 is considered the main bioactive product of MTX metabolism (Mewes et al. 1993; Panousis et al. 1997). We showed for the first time that this metabolite can be found in rat liver and heart, prolonging the exposure period of cardiac and hepatic cells to this bioactive compound. As demonstrated in vitro, the presence of MTX metabolites enhances the toxicity in a cardiomyoblast model. Furthermore, it was proved that the bioactivation of MTX mediated through CYP450 and namely CYP2E1 metabolism that occurs in loco in the cardiomyoblasts exerts a significant role in the cellular damage promoted by MTX. Thus, these results suggest that besides the relevance of MTX metabolism to its pharmacological action, the biotransformation fate of MTX might also be involved in its cardiotoxicity.

Acknowledgments Authors are grateful to Dr. Zelia dos Santos Azevedo, from Faculty of Sciences, University of Porto, for gently lend us the LC/DAD-ESI/MS and for all precious technical assistance. This work was supported by the Fundação para a Ciência e Tecnologia (FCT)—project [EXPL/DTP-FTO/0290/2012]-QREN initiative with EU/FEDER financing through COMPETE-Operational Programme for Competitiveness Factors. LGR and VMC thank FCT for their Ph.D. grant (SFRH/BD/63473/2009) and Post-doc grant (SFRH/BPD/63746/2009), respectively. The authors are also grateful to Fundação para a Ciência e a Tecnologia (FCT) for Grant No. PEstC/EQB/LA0006/2011

\section{References}

An G, Morris ME (2010) HPLC analysis of mitoxantrone in mouse plasma and tissues: application in a pharmacokinetic study. J Pharm Biomed Anal 51(3):750-753 
Avasarala JR et al (2003) Rapid onset mitoxantrone-induced cardiotoxicity in secondary progressive multiple sclerosis. Mult Scler 9:59-62

Basra J et al (1985) Evidence for human liver mediated-free radical formation by doxorubicin and mitozantrone. Anticancer Drug Des 1:45-52

Batra VK et al (1986) Pharmacokinetics of mitoxantrone in man and laboratory animals. Drug Metab Rev 17:311-329

Blanz J et al (1991) Evidence for oxidative activation of mitoxantrone in human, pig, and rat. Drug Metab Dispos 19(5):871-880

Brandon EF et al (2003) An update on in vitro test methods in human hepatic drug biotransformation research: pros and cons. Toxicol Appl Pharmacol 189(3):233-246

Brück TB, Brück DW (2011) Oxidative metabolism of the anti-cancer agent mitoxantrone by horseradish, lacto-and lignin peroxidase. Biochimie 93(2):217-226

Butler J, Hoey BM (1987) Are reduced quinones necessarily involved in the antitumour activity of quinone drugs? $\mathrm{Br} \mathrm{J}$ Cancer 8:53-59

Canal P et al (1993) Plasma and cellular pharmacokinetics of mitoxantrone in high-dose chemotherapeutic regimen for refractory lymphomas. Cancer Res 53(20):4850-4854

Duthie SJ, Grant MH (1989) The role of reductive and oxidative metabolism in the toxicity of mitoxantrone, adriamycin and menadione in human liver derived Hep G2 hepatoma cells. Br J Cancer 60(4):566-571

Ehninger $G$ et al (1990) Pharmacokinetics and metabolism of mitoxantrone. A review. Clin Pharmacokinet 18(5):365-380

Feofanov A et al (1997) Quantitative confocal spectral imaging analysis of mitoxantrone within living K562 cells: intracellular accumulation and distribution of monomers, aggregates, naphtoquinoxaline metabolite, and drug-target complexes. Biophys $\mathbf{J}$ 73(6):3328-3336

Fisher GR, Patterson LH (1992) Lack of involvement of reactive oxygen in the cytotoxicity of mitoxantrone, CI941 and ametantrone in MCF-7 cells: comparison with doxorubicin. Cancer Chemother Pharmacol 30(6):451-458

Fisher G, Patterson LH, Gutierrez PL (1993) A comparison of free radical formation by quinone anti-tumour agents in MCF-7 cells and the role of $\mathrm{NAD}(\mathrm{P}) \mathrm{H}$ (quinone-acceptor) oxidoreductase (DT-diaphorase). Chem Biol Interact 88:137-153

Hsin L-W et al (2008) Synthesis, DNA binding, and cytotoxicity of 1,4-bis(2-amino-ethylamino)anthraquinone-amino acid conjugates. Bioorg Med Chem 16(2):1006-1014

Jia L, Liu X (2007) The conduct of drug metabolism studies considered good practice (II): in vitro experiments. Curr Drug Metab 8(8):822-829

Khan SN et al (2010) Effect of mitoxantrone on proliferation dynamics and cell cycle progression. Biosci Rep 30(6):375-381

Kostrzewa-Nowak D et al (2007) Bioreductive activation of mitoxantrone by NADPH cytochrome P-450 reductase. Implications for increasing its ability to inhibit the growth of sensitive and multidrug resistant leukaemia HL60 cells. Cancer Lett 245(1-2): $252-262$
Li SJ, Rodgers EH, Grant MH (1995) The activity of xenobiotic enzymes and the cytotoxicity of mitoxantrone in MCF 7 human breast cancer cells treated with inducing agents. Chem Biol Interact 97(2):101-118

Lowry OH, Rosebrough NJ, Farr AL, Randall RJ (1951) Protein measurement with the folin phenol reagent, J Biol Chem (193): $265-272$

Mewes K et al (1993) Cytochrome P-450-induced cytotoxicity of mitoxantrone by formation of electrophilic intermediates. Cancer Res 53(21):5135-5142

Neuhaus O, Kieseier BC, Hartung H-P (2006) Therapeutic role of mitoxantrone in multiple sclerosis. Pharmacol Ther 109(1-2): 198-209

Novak RF, Kharasch ED (1985) Mitoxantrone: propensity for free radical formation and lipid peroxidation-implications for cardiotoxicity. Invest New Drugs 3(2):95-99

Panousis C, Kettle AJ, Phillips DR (1997) Neutrophil-mediated activation of mitoxantrone to metabolites which form adducts with DNA. Cancer Lett 113(1-2):173-178

Parkinson A, Ogilvie B (2008) Biotransformation of xenobiotics. In: Klaassen C (ed) Casarett \& Doull's toxicology: the basic science of poisons. McGraw-Hill, New York, p 1331

Pontes $\mathrm{H}$ et al (2010) Metabolic interactions between ethanol and MDMA in primary cultured rat hepatocytes. Toxicology 270(2-3):150-157

Richard B et al (1991) Interspecies variability in mitoxantrone metabolism using primary cultures of hepatocytes isolated from rat, rabbit and humans. Biochem Pharmacol 41(2):255-262

Rossato LG et al (2011) Structural isomerization of synephrine influences its uptake and ensuing glutathione depletion in ratisolated cardiomyocytes. Arch Toxicol 85(8):929-939

Sardão V et al (2009a) Doxorubicin-induced mitochondrial dysfunction is secondary to nuclear p53 activation in H9c2 cardiomyoblasts. Cancer Chemother Pharmacol 64(4):811-827

Sardão V et al (2009b) Morphological alterations induced by doxorubicin on H9c2 myoblasts: nuclear, mitochondrial, and cytoskeletal targets. Cell Biol Toxicol 25(3):227-243

Seiter K (2005) Toxicity of the topoisomerase II inhibitors. Expert Opin Drug Saf 4(2):219-234

Shipp NG et al (1993) Characterization of experimental mitoxantrone cardiotoxicity and its partial inhibition by ICRF- 187 in cultured neonatal rat heart cells. Cancer Res 53(3):550-556

Silva $\mathrm{R}$ et al (2011) In vitro study of P-glycoprotein induction as an antidotal pathway to prevent cytotoxicity in Caco-2 cells. Arch Toxicol 85(4):315-326

Wolf CR, Macpherson JS, Smyth JF (1986) Evidence for the metabolism of mitozantrone by microsomal glutathione transferases and 3-methylcholanthrene-inducible glucuronosyl transferases. Biochem Pharmacol 35(9):1577-1581

Yoshihara S et al (2001) Metabolic activation of bisphenol A by rat liver S9 fraction. Toxicol Sci 62(2):221-227

Zordoky BNM, El-Kadi AOS (2007) H9c2 cell line is a valuable in vitro model to study the drug metabolizing enzymes in the heart. J Pharmacol Toxicol Methods 56(3):317-322 